\title{
Ghost Images with Four-Mode Entangled States
}

\author{
A.V. Belinsky, P.P. Gostev and A.S. Chirkin ${ }^{\text {a }}$ \\ Faculty of Physics and International Laser Center, Moscow State University, 119991, Leninskie gory, Moscow, Russia
}

\begin{abstract}
We demonstrate that the use of multimode entangled states of light fields can restore multiple ghost images of the object illuminated by radiation of one of the modes. The analysis, performed on the example of four-mode entangled light fields, is able to obtain three copies of ghost images.
\end{abstract}

Nonclassical states of light fields allow to realize the so-called ghost images (GI) when the information about the object is not restored from his test beam. It is obtained in the presence of another, reference beam that is in a entangled state with the test one. Klyshko [1] for the first time drew attention on this opportunity. He showed that in such a situation the information about the object can be obtained from measurements of the spatial correlation function of photons of the test and references channels. Notice, the detection of radiation in the test channel does not give information about the object. GI research has been supported by the European project QUANTIM [2], and still continues to attract attention.

We first show that the application of multimode entangled states in the GI formation scheme allow us to create GI "copies". Carried out examination, using four-mode entangled states of light fields formed in coupled parametric processes, includes the process of spontaneous parametric downconversion and sum frequency generation. Thus we deal with the interactions of the following:

$$
\omega_{\mathrm{p}}=\omega_{1}+\omega_{2}, \omega_{\mathrm{p}}+\omega_{1}=\omega_{3}, \omega_{\mathrm{p}}+\omega_{2}=\omega_{4} \text {. }
$$

Quantum theory of these coupled processes and their application in quantum image are investigated in [3]. In [3] they are the basis of quantum holographic teleportation of entangled optical images. The calculations assume that the object is illuminated by radiation frequency and other frequency radiation directed to references channels. The radiation registered in the test channel does not provide information about the object. Under certain conditions, the information is restored from measurement of the spatial correlation function of the photon number fluctuations between the test photons and reference ones. Therefore we can simultaneously obtain three GI. Note that the radiation in all channels have different frequencies, and the spatial photon correlation function depends on transmission spatial-frequency coefficients connected with parametric processes (1).

This work was partially supported by the RFBR grants №13-07-00938, 14-02-00458.

\section{References}

1. D.N. Klyshko Sov. Phys. JETP 67, 1131 (1988)

2. Quantum images, edited by M.I. Kolobov (Springer, 2007)

3. M. Yu. Saygin, A.S. Chirkin, M.I. Kolobov, J. Opt. Soc. Am. B 29, 2090 (2012)

\footnotetext{
${ }^{\mathrm{a}}$ Corresponding author: aschirkin@rambler.ru
} 